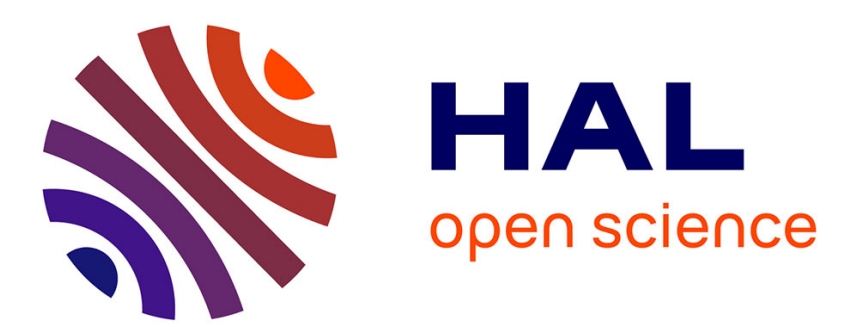

\title{
Cell cycle period control through modulation of clock inputs
}

Sofia Jose Figueiredo Almeida, Madalena Chaves, Franck Delaunay

\section{To cite this version:}

Sofia Jose Figueiredo Almeida, Madalena Chaves, Franck Delaunay. Cell cycle period control through modulation of clock inputs. Journal of Bioinformatics and Computational Biology, 2020, 18 (03), pp.2040006. 10.1142/S0219720020400065 . hal-03105546

\section{HAL Id: hal-03105546 \\ https://hal.inria.fr/hal-03105546}

Submitted on 11 Jan 2021

HAL is a multi-disciplinary open access archive for the deposit and dissemination of scientific research documents, whether they are published or not. The documents may come from teaching and research institutions in France or abroad, or from public or private research centers.
L'archive ouverte pluridisciplinaire HAL, est destinée au dépôt et à la diffusion de documents scientifiques de niveau recherche, publiés ou non, émanant des établissements d'enseignement et de recherche français ou étrangers, des laboratoires publics ou privés. 
Journal of Bioinformatics and Computational Biology

(C) Imperial College Press

\title{
Cell cycle period control through modulation of clock inputs
}

\author{
S. ALMEIDA \\ Inria, iBV, Université Côte d'Azur \\ Sophia Antipolis \\ France \\ sofia.jf.almeida@gmail.com \\ M. CHAVES* \\ Inria, Université Côte d'Azur, INRA, CNRS, Sorbonne Université \\ Sophia Antipolis \\ France \\ madalena.chaves@inria.fr \\ F. DELAUNAY ${ }^{\dagger}$ \\ iBV, Université Côte d'Azur, CNRS, INSERM \\ Sophia Antipolis \\ France \\ Franck.Delaunay@univ-cotedazur.fr
}

Received (Day Month Year)

Revised (Day Month Year)

Accepted (Day Month Year)

\begin{abstract}
In this work we study period control of the mammalian cell cycle via coupling with the cellular clock. For this, we make use of the oscillators' synchronization dynamics and investigate methods of slowing down the cell cycle with the use of clock inputs. Clock control of the cell cycle is well established via identified molecular mechanisms, such as the CLOCK:BMAL1-mediated induction of the wee1 gene, resulting in the WEE1 kinase that represses the active form of MPF (mitosis promoting factor), the essential cell cycle component. To investigate the coupling dynamics of these systems, we use previously developed models of the clock and cell cycle oscillators and center our studies on unidirectional clock $\rightarrow$ cell cycle coupling. Moreover, we propose an hypothesis of a Growth Factor (GF)-responsive clock, involving a pathway of the non-essential cell cycle complex cyclin D/CDK4. We observe a variety of rational ratios of clock to cell cycle period, such as: 1:1, 3:2, 4:3 and 5:4. Finally, our protocols of period control are successful in effectively slowing down the cell cycle by the use of clock modulating inputs, some of which correspond to existing drugs.
\end{abstract}

Keywords: Modeling; Synchronization; Circadian Clock; Cell Cycle; Period Control

*http://www-sop.inria.fr/members/Madalena.Chaves/

${ }^{\dagger}$ http://ibv.unice.fr/research-team/delaunay/ 


\section{Introduction}

The mammalian cell cycle is a cyclic process of cellular growth and renewal that culminates in mitosis, where a mother cell divides into two daughter cells. An unregulated cell cycle is a characteristic of cancer, that may arise due to mutations inducing accelerated and/or uncontrolled cellular division. Thus, methods of cell cycle control are a current topic of important research focus. In this work we study the unidirectional clock $\rightarrow$ cell cycle coupling with special emphasis on developing methods of cell cycle period control with the use of clock regulators.

The circadian cell clock coordinates $24 \mathrm{~h}$ rhythms of gene activation and protein expression, allowing the adaption of cells to daily changes in external conditions. It plays a major role in cellular control, with genome-wide studies revealing circadian patterns of control for a large proportion of drug targets ${ }^{1}$. Moreover, deregulation of circadian rhythms may result in insulin resistance and inflammation ${ }^{2}$.

The mammalian clock and cell cycle are often synchronized in healthy cells and molecular interactions denoting an action of the clock on the cell cycle have been identified. These interactions consist in the clock-controlled repression of c-Myc ${ }^{3}$, a promoter of cell cycle progression via cyclin E induction ${ }^{4}$, the regulation of the cell cycle inhibitor p21 by REV-ERB- $\alpha / \beta$ and $\operatorname{ROR}-\alpha / \gamma^{5}$, which are a part of the clock, and the induction of the MPF repressor wee1 by the CLOCK:BMAL1 complex ${ }^{6}$. The cell cycle interconnection with the clock may be further evidenced by the joint association of cancer with circadian clock disturbances, inflammation and abnormal cellular metabolism ${ }^{7}$. Furthermore, it has been established that chronotherapy - the timed application of drugs - both maximizes the desired drug effect and minimizes undesired side-effects ${ }^{1}$.

In addition, evidence of a possible cell cycle action on the clock has also been discovered. This observation, made by Feillet et al. ${ }^{8}$ and Bieler et al. ${ }^{9}$, revealed the joint acceleration of the clock and cell cycle with the increase of \% of FBS (Fetal Bovine Serum) in NIH3T3 mouse fibroblasts. FBS is added to the medium and consists of Growth Factors (GFs) that are known to accelerate the cell cycle. As the cell cycle accelerates with Growth Factor, so does the clock, such that oscillation periods remain in a 1:1 ratio. These observations $\left({ }^{8,9}\right)$ gave rise to an hypothesis of an action of the cell cycle on the clock, suggesting that the oscillators may be bidirectionally coupled.

Furthermore, a pulse of Dexamethasone (Dex) results in different synchronization ratios depending on FBS concentration ${ }^{8}$. Dex is a glucocorticoid agonist known to induce and synchronize the clocks of cell populations. For $10 \%$ FBS, Dex-treated cells presented approximately a 5:4 synchronization ratio of clock to cell cycle period. For 20\% FBS, Dex-treated cells segregate in two groups: one maintaining 1:1 synchronization and the other showing a 3:2 synchronization ratio. Almeida et al.

${ }^{10}$ have reproduced the Dex-treatment protocol in silico replicating the induction of the 3:2 ratio in both unidirectional cell cycle $\rightarrow$ clock and bidirectional coupling.

Additionally, cellular division is observed to occur at a specific clock phase for 
all cells ${ }^{8}$, which is consistent with a model of oscillators that are phase-locked. Phase locking is characterized by convergence of the combined phase of oscillation $\phi(t)=\left(\phi_{1}(t), \phi_{2}(t)\right)$ to a closed curve - an attractor, and phase-locked oscillators are synchronized through the entire cycle. In Dex-treated cells a trimodal peak occurs for the distribution of mitosis with circadian clock phase ${ }^{8}$, an observation that has also been made by Nagoshi et al. ${ }^{11}$.

Our work is an extension of Almeida et al. ${ }^{12}$ and aims at exploring strategies of cell cycle period control in unidirectional clock $\rightarrow$ cell cycle coupling. For this, we use previously developed dynamical models of both systems (see Section 2). Thus, in Section 3, we model the CLOCK:BMAL1-induced repression of MPF via the wee1 pathway. Other models describing an action of the clock on the cell cycle are for instance provided by Zámborszky et al. ${ }^{13}$ and Gérard and Goldbeter ${ }^{14}$, while studies of molecular interactions of cell cycle $\rightarrow$ clock coupling were made by Traynard et al. ${ }^{15}$, Almeida et al. ${ }^{10}$ and Yan and Goldbeter ${ }^{16}$. Here, we propose yet a novel hypothesis: that of a clock that is responsive to Growth Factor (GF). This is because unidirectional coupling can be sufficient for 1:1 phase-lock and observations show the increase of both clock and cell cycle frequencies with GF. As such, in Section 4 we model the GF/clock interaction based on a molecular pathway of cyclinD/CDK4 and PGC1- $\alpha{ }^{17}$.

Throughout the work, we observe the phase-locked oscillator, but focus mostly on observing variations of clock to cell cycle periods ratios, i.e. on the system's period-lock (or synchronization) state. We obtain 1:1, 3:2, 4:3 and 5:4 ratios of clock to cell cycle period. From the knowledge gained from the system's synchronization dynamics, we devise strategies of period control, with the goal of slowing down the cell cycle. We are able to obtain higher cell cycle periods via two main strategies: by slowing down the clock maintaining 1:1 synchronization and by inducing synchronization states of 1:3, 1:2, 2:3, 3:4 and 4:5 clock to cell cycle period ratios. Our methods provide an insight of multiple ways of cell cycle period control relying on the dynamics of its synchronization with the circadian cell clock.

\section{Methods}

The circadian clock model was developed by Almeida et al. ${ }^{18}$ and reduced by Almeida et al. ${ }^{10}$ and is centered on the regulation occurring at three types of DNA response elements within gene promoters: E-boxes, R-boxes and D-boxes. It describes the main transcription translation feedback loop of the mammalian cell clock, in which the CLOCK:BMAL1 protein complex promotes expression of the PER and CRY proteins that may then form the PER:CRY complex, a repressor of CLOCK:BMAL1. It has been reduced to 4 variables: BMAL1, REV, DBP and PER:CRY. Here, BMAL1 represents the CLOCK:BMAL1 complex, REV is all REV-ERBs and DBP represents a general D-box activator.

The cell cycle model was developed and analyzed by Almeida et al. ${ }^{19}$ and in its most reduced form consists of a two variable system forming a main negative 
regulatory loop between the essential cell cycle component MPF (mitosis promoting factor), which is the cyclin B/CDK1 complex, necessary and sufficient to carry out the mitotic process, and its repressor the APC:cdc20 complex. This model includes terms describing the action of CDC25 phosphatase, an MPF activator, and the WEE1 kinase, an MPF repressor ${ }^{19}$.

The model equations are:

$$
\begin{gathered}
\frac{d[B M A L 1]}{d t}=R_{b o x}-\gamma_{b p}[B M A L 1][P E R: C R Y] \\
\frac{d[D B P]}{d t}=V_{B}[B M A L 1]-\gamma_{d b}[D B P] \\
\frac{d[R E V]}{d t}=V_{D 2}[D B P]-\gamma_{r e v}[R E V] \\
\frac{d[P E R: C R Y]}{d t}=D e x+V_{D 2}[D B P]-\gamma_{b p}[B M A L 1][P E R: C R Y] \\
\frac{d[M P F]}{d t}=G F+V_{c} \frac{\frac{M P F_{\max }}{M P F_{\max }}-[M P F]+k_{c}}{[M P F]^{2}+k_{m}^{2}} \\
-V_{w} \frac{[M P F]}{[M P F]+k_{w}} \frac{k_{n}^{2}}{[M P F]^{2}+k_{n}^{2}} \\
-\gamma_{1}[A P C: c d c 20][M P F] \\
\frac{d[A P C: c d c 20]}{d t}=V_{m}[M P F]-V_{k}[A P C: c d c 20]
\end{gathered}
$$

where

$$
R_{b o x}=V_{R} \frac{k_{R r}^{2}}{k_{R r}^{2}+[R E V]^{2}}
$$

and the parameter GF represents an MPF synthesis term due to the presence of Growth Factor. The Dex additive input to PER:CRY models the Dex-treatment used in Section 4 and is kept at zero for the remaining studies.

These models have been used by Almeida et al. ${ }^{10}$ to study cell cycle $\rightarrow$ clock coupling as well as bidirectional coupling. The initial condition used is: BMAL = $1.2 ; \mathrm{DBP}=1.6 ; \mathrm{REV}=1.5 ; \mathrm{PER}: \mathrm{CRY}=1.2 ; \mathrm{MPF}=2.0 ; \mathrm{APC}: \operatorname{cdc} 20=1.0 ;$ parameters are shown in Table 1.

\subsection{Modeling coupling mechanisms}

Firstly, we model unidirectional clock $\rightarrow$ cell cycle coupling via the induction of the MPF repressor wee1 by CLOCK:BMAL1. The action of WEE1 on MPF is included in the term $V_{w}{ }^{19}$. Thus, we multiply this term by the variable BMAL1 to represent the repressor effect of CLOCK:BMAL1. The equation for MPF becomes: 
Table 1. Parameters of the clock and cell cycle models

Cell Cycle

\begin{tabular}{cccccccccc}
\hline$V_{c}\left(h^{-1}\right)$ & $k_{c}$ & $V_{w}\left(h^{-1}\right)$ & $k_{w}$ & $V_{m}\left(h^{-1}\right)$ & $V_{k}\left(h^{-1}\right)$ & $\gamma_{1}\left(h^{-1}\right)$ & $k_{m}$ & $k_{n}$ & $\overline{M P F_{\max }}$ \\
\hline 2260 & 130 & 7480 & 138 & 0.168 & 0.107 & 0.162 & 99 & 0.116 & 284 \\
\hline \multicolumn{8}{c}{ Circadian } & Clock \\
\hline$V_{R}\left(h^{-1}\right)$ & $k_{R r}$ & $V_{B}\left(h^{-1}\right)$ & $\gamma_{b p}(h)$ & $V_{D 2}\left(h^{-1}\right)$ & $\gamma_{\text {rev }}\left(h^{-1}\right)$ & $\gamma_{d b}\left(h^{-1}\right)$ \\
\hline 34.4 & 80.1 & 0.11 & 2.0 & 14.7 & 0.187 & 0.121 \\
\hline
\end{tabular}

$$
\begin{aligned}
\frac{d[M P F]}{d t}= & G F+V_{c} \frac{\overline{M P F_{\max }}-[M P F]}{\overline{M P F_{\max }}-[M P F]+k_{c}} \frac{[M P F]^{2}}{[M P F]^{2}+k_{m}^{2}} \\
& -\mathbf{c}_{\mathbf{b}}[\mathbf{B M A L 1}] V_{w} \frac{[M P F]}{[M P F]+k_{w}} \frac{k_{n}^{2}}{[M P F]^{2}+k_{n}^{2}} \\
& -\gamma_{1}[A P C: c d c 20][M P F]
\end{aligned}
$$

where $c_{b}$ is the coupling strength parameter.

Moreover, we propose a different additional mechanism with possible relevance for clock and cell cycle coupling. Experimental observations show that increasing GF increases the frequency of both cell cycle and clock in 1:1 synchronization ${ }^{8}$. Thus, we study GF as a common input to both oscillators. For this we consider a potential pathway connecting GF with the clock.

Growth factors promote expression of cyclin $\mathrm{D}$ that is active when in a complex with either CDK4 or CDK6, via $\beta$-catenin mediated pathways ${ }^{20}$. The action of cyclin D in the G1 phase precedes the activation of subsequent cyclin-CDK complexes. Nevertheless, cyclin D is approximately constant during the cell cycle and is non-essential, as its deletion mutants still can undergo mitosis. Therefore, considering cells can't divide without GF, there must be other pathways of GF action on the essential cell cycle elements, namely the cyclin B-CDK1 complex (MPF), independently of cyclind D. Thus, a pathway connecting GF $\rightarrow$ cyclin D-CDK4/CDK6 $\rightarrow$ clock is independent of the essential cell cycle components.

The cyclin D-CDK4 complex regulates PGC1- $\alpha$, by promoting its repressor GCN5 ${ }^{17}$ (also known as KAT2A). PGC1- $\alpha$ is an important clock component that promotes binding of ROR to R-box. As such, by promoting cyclin D expression, GF indirectly represses PGC1- $\alpha$ and therefore R-box. This effect can be introduced by making the change $V_{R} \rightarrow V_{R} \frac{k_{s}}{k_{s}+G F}$. R-box now becomes:

$$
R_{b o x}=V_{R}\left(\frac{k_{s}}{k_{s}+G F}\right) \frac{k_{R r}^{2}}{k_{R r}^{2}+[R E V]^{2}}
$$


Fig. 1 shows a scheme of the system in simple unidirectional clock $\rightarrow$ cell cycle coupling (A) and with the same mechanism under a GF-responsive clock (B).

A)

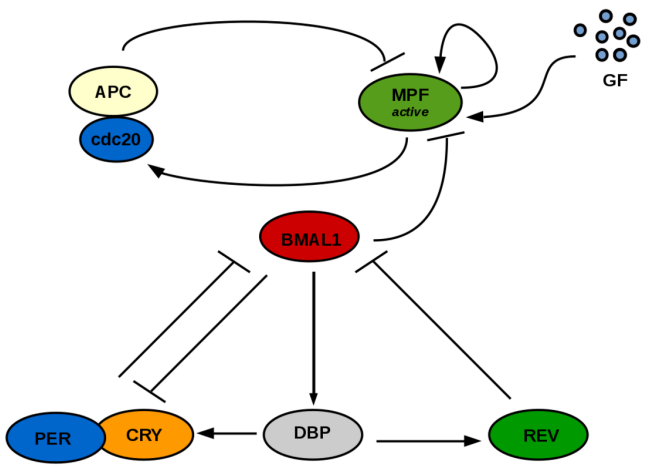

B)

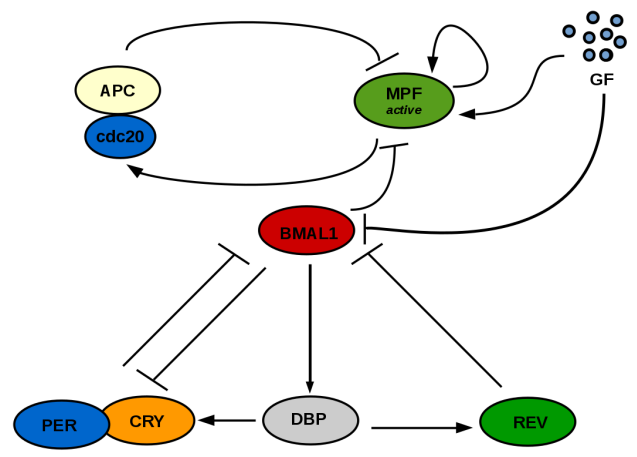

Fig. 1. Schematics of the two coupling mechanisms. A) Clock $\rightarrow$ cell cycle coupling: BMAL1 represses $M P F_{\text {active }}$ due to its action in promoting expression of the wee1 gene. B) Clock $\rightarrow$ cell cycle coupling with a GF-responsive clock: the previous coupling mechanism is maintained and now GF represses R-box (at the BMAL1 promoter) via the cyclin D-CDK4/PGC1 $1_{\alpha}$ pathway.

\section{Unidirectional Clock $\rightarrow$ Cell Cycle Coupling}

Fig. 2 shows a solution of MPF and BMAL1 using the model developed on the previous Section (Fig $1 \mathrm{~A}$ and Equation 8). For GF $=10$ and $c_{b}=10$ the clock entrains the cell cycle to its intrinsic period of $24 \mathrm{~h}$. Because both clock and cell cycle systems were previously normalized to a certain concentration value ${ }^{18}{ }^{19}$, the solution of the coupled system is dimensionless.

Now, we observe how control parameters affect the synchronization state. Fig. 3 shows the effect of GF on synchronization ratios for fixed $c_{b}=10$ and $c_{b}=100$. The ratio of clock to cell cycle period $\left(r_{T}=\frac{T_{\text {clock }}}{T_{\text {cell }} \text { cycle }}\right)$ tends to increase with GF. Most notably with $c_{b}=100$, synchronization ratios are constant by intervals of GF, assuming a rational value in this interval. This pattern is the devil's staircase ${ }^{21}$.

Furthermore, Fig. 4 shows the periods of both oscillators for $c_{b}=10$ and $c_{b}$ $=100$ for the same simulations as Fig. 3. Note that, as expected for the clock $\rightarrow$ cell cycle unidirectional coupling, the period of the clock is kept at $24 \mathrm{~h}$, while the period of the cell cycle is sped up in response to GF. Thus, regions of a slower clock than cell cycle appear. This in accordance with experimental observations ${ }^{8}$.

Moreover, the system's synchronization response to variations of the control parameter $c_{b}$ is shown in Table 2 for fixed GF. Ratios $r_{T}$ of 4:3 and 5:3 are now present. $\mathrm{GF}=20$ results in 4:3 and 1:1 synchronization, while $\mathrm{GF}=40$ drives the system to 5:3, 3:2 and 1:1 synchronization states. Additionally, GF $=20$ requires a lower value of $c_{b}$ than $\mathrm{GF}=40$ to induce the 1:1 period-lock. 


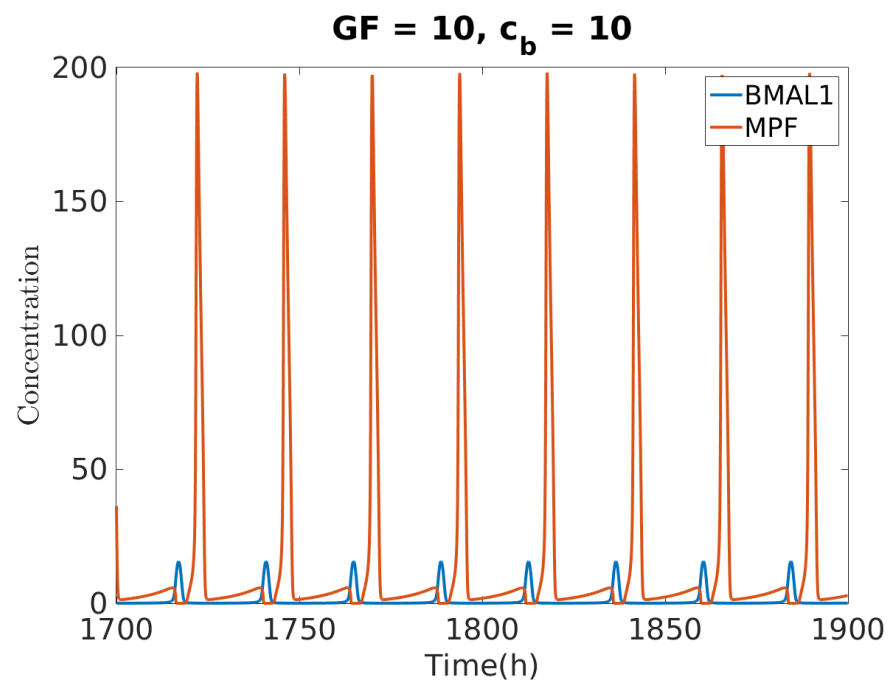

Fig. 2. Oscillation of MPF and BMAL1. The system oscillates with a $24 \mathrm{~h}$ period.
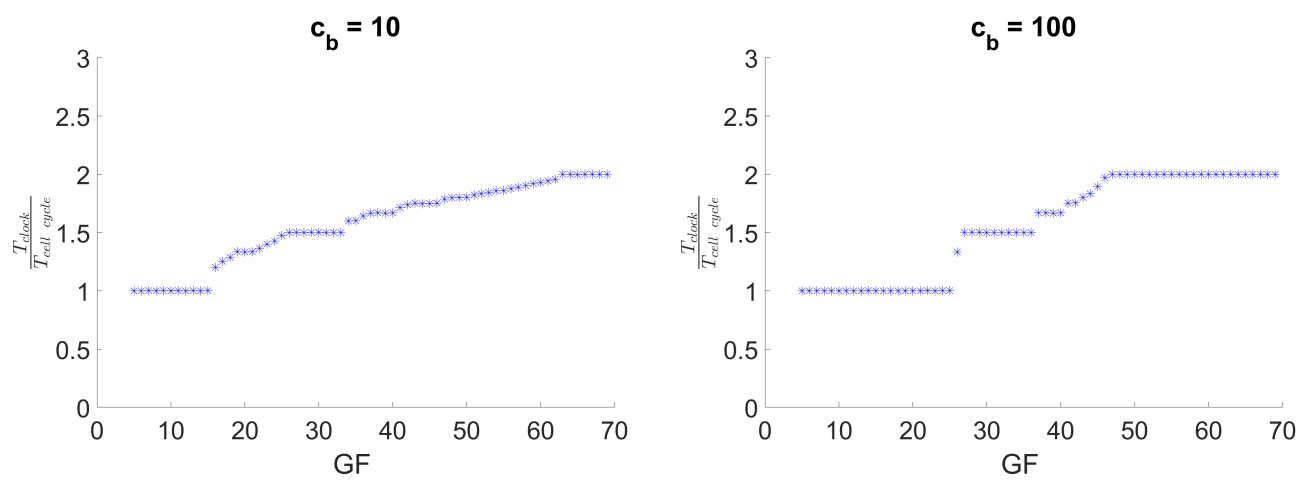

Fig. 3. Variation of the clock to cell cycle synchronization ratio with $G F$ for $c_{b}=10$ and $\mathbf{c}_{\mathbf{b}}=100 . r_{T}$ is constant by intervals of GF, in a devil's staircase-like pattern.

A question of interest is the possibility of slowing down the cell cycle using the dynamics of the coupled system. From Fig. 4, this may result from slowing down the clock together with coincidental period ratios $\left(r_{T}=1\right)$. On the other hand, because we have observed $r_{T}>1$, the question of being able to induce $r_{T}<1$ is raised. This hypothesis, if proven, would represent an adaptation of the cell cycle period to a higher value than that of the clock period. 

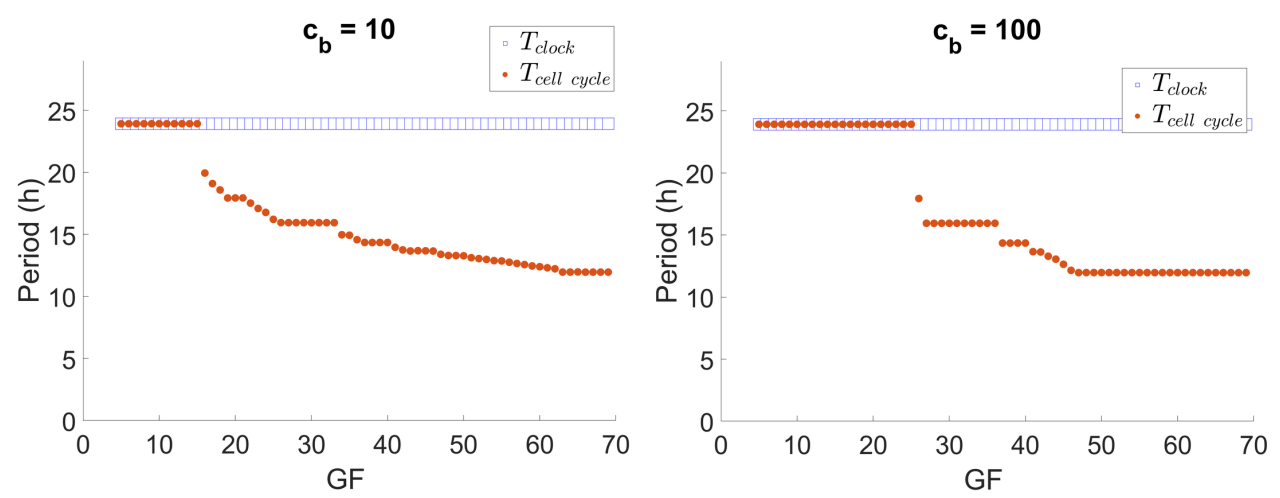

Fig. 4. Circadian clock and cell cycle periods for $c_{b}=10$ and $c_{b}=100$.

Table 2. Coupling strength $c_{b}$ controls the system's synchronization ratio

\begin{tabular}{cccccccccccccccc}
\multicolumn{110}{c}{$\mathbf{G F}=$} & $\mathbf{2 0}$ \\
\hline $\mathbf{c}_{\mathbf{b}}$ & 4 & 8 & 12 & 16 & 20 & 24 & 28 & 32 & 36 & 40 & 44 & 48 & 52 & 54 \\
\hline $\mathbf{r}_{\mathbf{T}}$ & 1.33 & 1.33 & 1.33 & 1.33 & 1.33 & 1.33 & 1.33 & 1.33 & 1.33 & 1.31 & 1.00 & 1.00 & 1.00 & 1.00 \\
\hline \multicolumn{11}{c}{10} \\
\hline $\mathbf{c}_{\mathbf{b}}$ & 40 & 60 & 80 & 100 & 120 & 140 & 160 & 180 & 200 & 220 & 240 & 260 & 280 & 300 \\
\hline $\mathbf{r}_{\mathbf{T}}$ & 1.67 & 1.67 & 1.67 & 1.67 & 1.67 & 1.67 & 1.67 & 1.67 & 1.67 & 1.67 & 1.50 & 1.50 & 1.00 & 1.00 \\
\hline
\end{tabular}

\subsection{Cell Cycle Period Control with Clock Inputs}

The parameters that affect clock period the most are those of R-box $\left(V_{R}\right.$ and $\left.k_{R r}\right)$ as well as the rates of REV and DBP natural degradation $\left(\gamma_{r e v}\right.$ and $\left.\gamma_{d b}\right)$, see Almeida et al. ${ }^{18}$. Thus, we first introduce the parameter $\alpha$ in the R-box equation as: $R_{b o x}=V_{R} \frac{k_{R r}^{2}}{k_{R r}^{2}+(\alpha[R E V])^{2}}$, which is identical to rescaling the parameter $k_{R r} \rightarrow \frac{k_{R r}}{\alpha}$. Here, the parameter $\alpha$ can either represent an R-box agonist (REV antagonist) for $\alpha<1$ or an R-box antagonist (REV agonist) for $\alpha>1$, in relation to the control state $\alpha=1$ oscillating with the intrinsic period.

Fig. 5 shows the period response of both oscillators with $\alpha$ and Fig. 6 the synchronization ratio response. Observe that increasing $\alpha$ increases the periods of both clock and cell cycle in 1:1 synchronization. On the other hand, for small values $\alpha$ there is a region where a smaller clock period is combined with a substantially increase in cell cycle period, in accordance with our hypothesis.

Now, we study period control by tuning of the REV and DBP rates of degrada- 


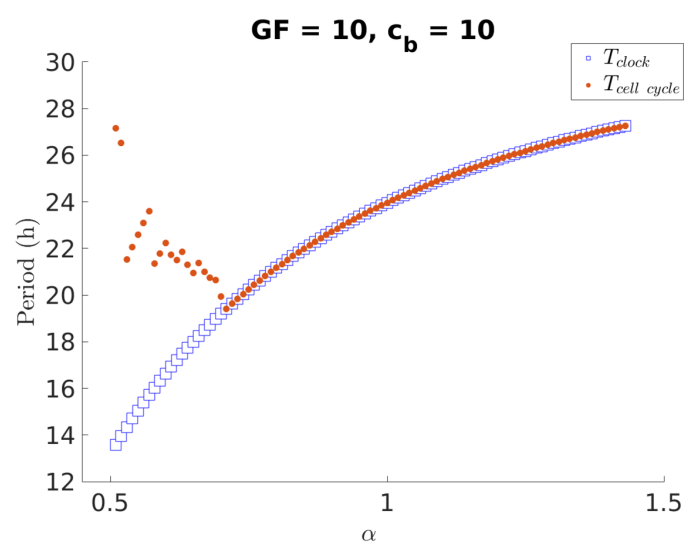

Fig. 5. Evolution of the oscillators' period with $\alpha$.

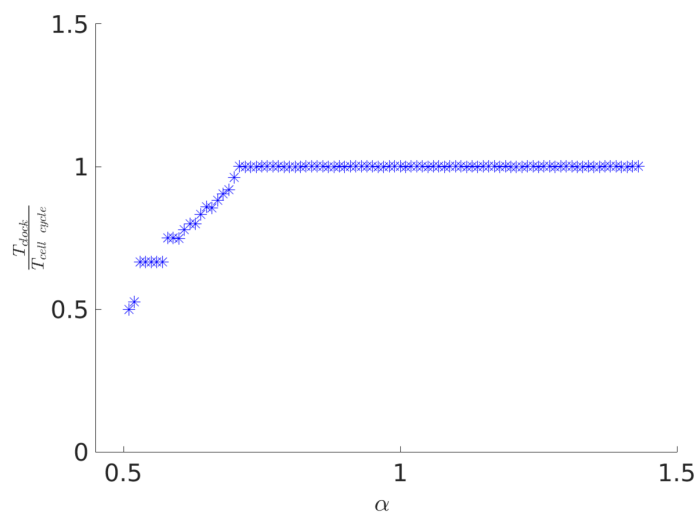

Fig. 6. Evolution of the oscillators' synchronization ratio with $\alpha$. Overall, the $1: 1,1: 2,2: 3$, $3: 4$ and 4:5 ratios are obtained.

tion $\gamma_{r e v}$ and $\gamma_{b p}$. For this, we introduce $\beta$ and $\delta$ as modulators of these parameters as: $\gamma_{r e v} \rightarrow \beta \gamma_{r e v}$ and $\gamma_{d b} \rightarrow \delta \gamma_{d b}$. Fig. 7 , shows a solution of the system with a combination of increased $\gamma_{r e v}$ and $\gamma_{d b}$. In this case, $\beta=2$ and $\delta=1.5$ leads to a much slower cell cycle overall $(\mathrm{T}=31.5 \mathrm{~h})$ and a $1: 3$ period entrainment.

Some available compounds are known to interfere with $\gamma_{\text {rev }}$ that could thus be used for tuning this parameter. These drugs act mostly via inhibition of GSk3 $\beta$, known to increase phosphorylation of REV-ERB, and can lead either to a decreased or increased clock period ${ }^{22}$ depending on the GSK-inhibitor used ${ }^{23} 24$.

Note that healthy cells are likely to be in $1: 1$ synchronization ${ }^{25}$. Thus, experimental exploration of chemical compounds for the increase of $c_{b}$, which promotes 1:1 synchronization, would also be relevant. 


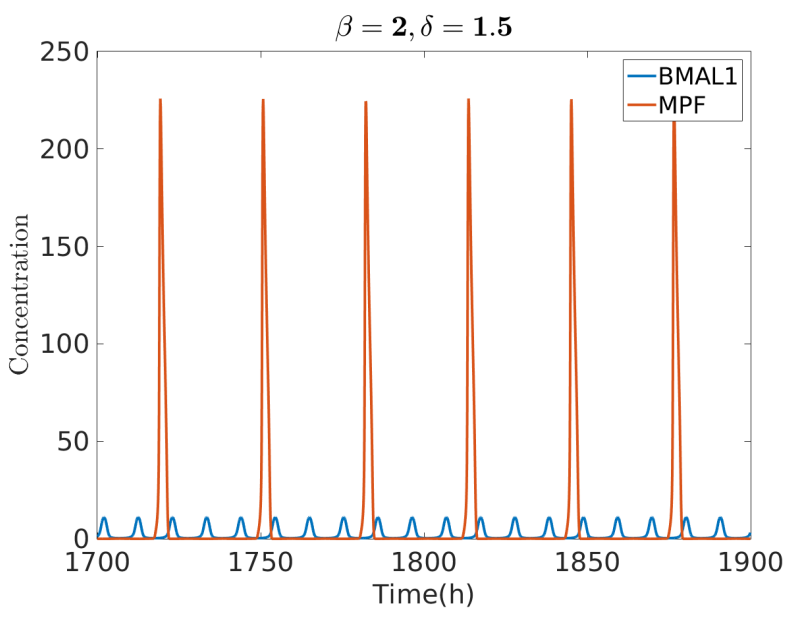

Fig. 7. Oscillation of clock and cell cycle for $\beta=\mathbf{2}, \delta=\mathbf{1 . 5}$, with $\mathbf{G F}=\mathbf{1 0}$ and $\mathbf{c}_{\mathbf{b}}=\mathbf{1 0}$. The system synchronizes with a 1:3 ratio: $T_{\text {clock }}=10.5 \mathrm{~h}$ and $T_{\text {cell cycle }}=31.5 \mathrm{~h}$.

\section{Coupling with a GF-induced Clock}

Using the model developed on Section 2.1 for a GF-responsive clock (Fig $1 \mathrm{~B}$ and Equations 8 and 9), we analyze the effect of GF on the oscillators' synchronization state for fixed $c_{b}$ with and without a Dex input. This model assumes GF as a clock input that can control its period but it's still not required for oscillation. As such, $\mathrm{GF}=0$ yields the $24 \mathrm{~h}$ intrinsic clock oscillation that has been the basis of our clock studies so far. Furthermore, we verify that with this model (with $k_{s}=10$ ) the clock is accelerated with GF and clock oscillations are maintained in the entire GF region of cell cycle oscillation $(4 \leq \mathrm{GF} \leq 80)$.

Fig. 8 (left) shows that for $c_{b}=10$ a region of 1:1 period-lock is followed by a region where the ratio of clock to cell cycle period increases up to a value of 1.3. This synchronization ratio adequately compares with the experimental observations of Feillet et al. ${ }^{8}$. Moreover, a Dex additive input on the PER equation (see Equation 4 of Section 2) alters the system's behavior, resulting in a narrower region of 1:1 synchronization. The point of synchronization state change is shifted to a lower value of GF. This "shift to the left" effect evidences a GF region where the presence of Dex drives the system away from the 1:1 synchronization state (an example would be $\mathrm{GF}=20$ ), which is consistent with experimental observations ${ }^{8}$. The recovery of the induction of $r_{T}>1$ with Dex application was also achieved by Almeida et al. ${ }^{10}$ in unidirectional cell cycle $\rightarrow$ clock and bidirectional coupling, albeit in a different synchronization dynamic: a devil's staircase pattern of $r_{T}$ with GF, similar to what was observed in the previous Section.

Moreover, we investigate the local maximum of $r_{T}$ in the region where the system breaks away from 1:1 synchronization (see Fig. 8). Observe on Table 3 the maximum 

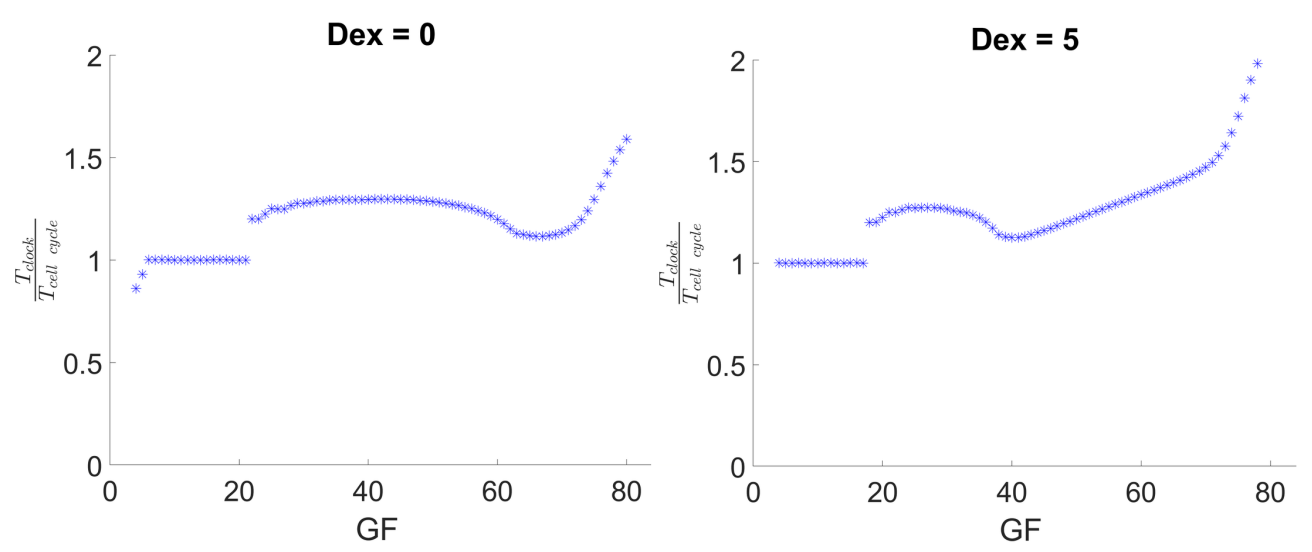

Fig. 8. Variation of $\mathbf{r}_{\mathbf{T}}$ with GF for $\mathbf{c}_{\mathbf{b}}=10$, with Dex $=0$ and Dex $=5$.

$r_{T}$ value for $4 \leq G F \leq 45$, for different values of $c_{b}$ with and without Dex. Without Dex, synchronization ratios vary between 5:4 and 4:3. With Dex the maximum $r_{T}$ value of the system is lower.

Table 3. Maximum synchronization ratio with $c_{b}$ for $4 \leq G F \leq 45$, with Dex $=0$ and Dex $=5$.

\begin{tabular}{ccccc}
$c_{b}$ & 10 & 20 & 30 & 40 \\
\hline Dex $=0$ & 1.30 & 1.33 & 1.33 & 1.25 \\
\hline Dex $=5$ & 1.28 & 1.29 & 1.25 & 1.18 \\
\hline
\end{tabular}

Additionally, Fig. 9 shows the variation of the oscillators' period with GF for $c_{b}=10$, with and without Dex (same simulation as Fig. 8). By contrast with Fig. 4, the period of the clock is now responsive to GF. Nevertheless, note that in the GF region where the ratio between periods is kept close to a constant there is an adaptation of the cell cycle period to that of the clock, as expected for the unidirectional clock $\rightarrow$ cell cycle coupling. Thus, this raises the question of the possibility of cell cycle period control via clock tuning.

As such, similarly to Section 3, we make $\gamma_{\text {rev }} \rightarrow \beta \gamma_{\text {rev }}$ and vary $\beta$ for values around 1, see Fig. 10. Observe that slowing down the clock by decreasing $\beta$ effectively slows down the cell cycle maintaining 1:1 synchronization. For $1 \leq \beta<1.4$ speeding up the clock results in a sped up cell cycle, as the oscillators maintain the 1:1 synchronization, and for $\beta \geq 1.4$ the system breaks out of the 1:1 synchronization state and states of $r_{T}<1$ appear again, where the cell cycle is slower than the clock and, after a certain $\beta$ value, than the control system (with $\beta=1$ ).

Finally, Fig. 11 shows a solution of the system with a combination of increased 

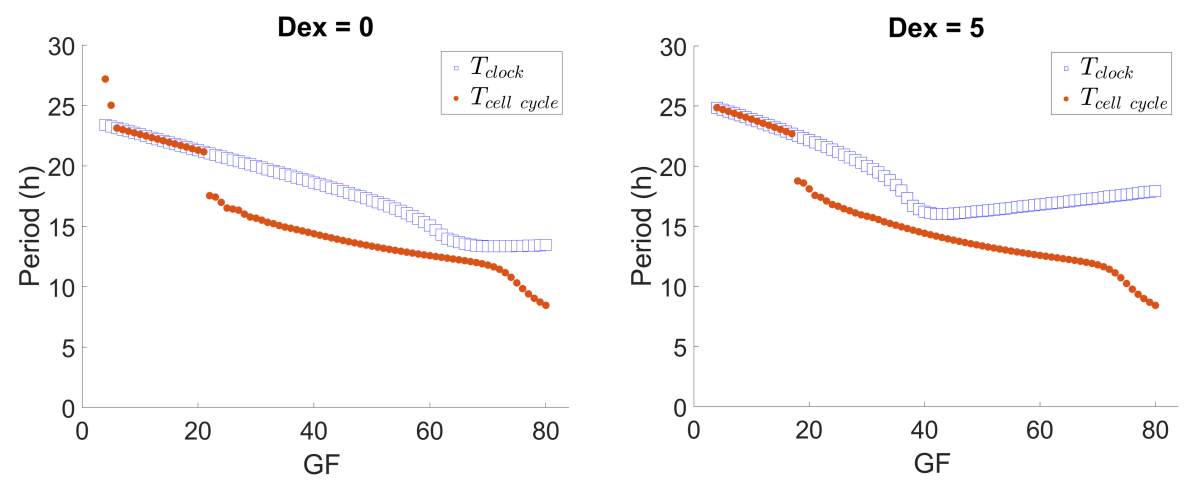

Fig. 9. Change in oscillators' period with GF for $c_{b}=10$, with and without Dex.

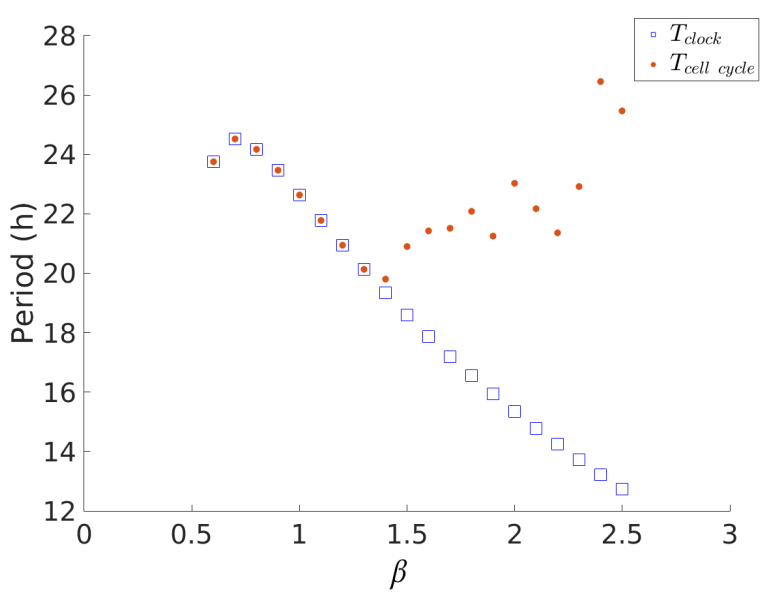

Fig. 10. Variation of the clock and cell cycle periods with $\beta$.

$\gamma_{\text {rev }}$ and $\gamma_{d b}$. In this case, $\beta=1.5$ and $\delta=1.3$ (defined as $\gamma_{d b} \rightarrow \delta \gamma_{d b}$ ) leads to $T_{\text {cell cycle }}=27 \mathrm{~h}$ and $T_{\text {clock }}=13.5 \mathrm{~h}$ in $1: 2$ synchronization, slowing down the cell cycle in relation to the control system with $\beta=1$ and $\delta=1$.

\section{Conclusion}

In this work, we have obtained ratios of clock to cell cycle period compatible with experimental observations ${ }^{8}$ under unidirectional clock $\rightarrow$ cell cycle coupling with and without a GF-responsive clock. Furthermore, Dex application results in inducing $r_{T}>1$, also in accordance with observations ${ }^{8}$.

In general, the hypothesis of a GF-responsive clock here proposed adds another layer of complexity to the coupling problem, as it raises the question of the rel- 

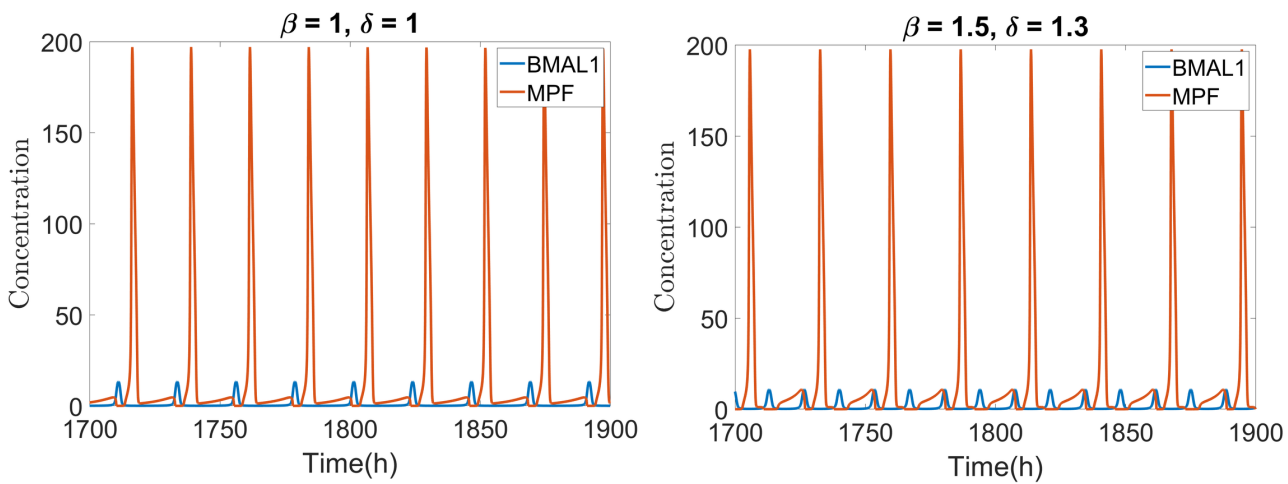

Fig. 11. Oscillation of MPF and BMAL1. For GF $=10$ and $c_{b}=10$ the system oscillates with a $22.6 \mathrm{~h}$ period in $1: 1$ phase-lock for $\beta=1$ and $\delta=1$. Making $\beta=1.5$ and $\delta=1.3$ drives the system to $1: 2$ phase-lock where $T_{\text {clock }}=13.5 \mathrm{~h}$ and $T_{\text {cell cycle }}=27 \mathrm{~h}$.

evance of GF-transducing pathways versus the cell cycle for circadian rhythms, and whether this may or not be influenced by the cellular (and extracellular) context (metabolism, signaling). In particular, the pathway proposed by us on Section 4 could be tested via knock-out experiments of the intermediary components cyclin D/CDK4 and GCN5. Nevertheless, this is an hypothesis difficult to address experimentally because it would be a challenge to test a GF effect on the clock independently of the cell cycle in dividing cells as, besides PGC1- $\alpha$, GCN5 interacts with several proteins involved in the clock mechanism such as WDR5, MYC and AKT1 that act on various cellular levels.

From a biological point of view, an exclusively unidirectional clock $\rightarrow$ cell cycle coupling mechanism implies a GF-responsive clock in order to verify the increase of clock's frequency with GF. A bidirectional coupling on the other hand requires modeling of cell cycle $\rightarrow$ clock molecular interactions, that have yet to be established. An action of MPF in phosphorylating an essential clock component is proposed by Almeida et al. ${ }^{10}$, a modeling choice that also yields synchronization ratios comparable to experimental observations. Nevertheless, these two hypothesis aren't mutually exclusive, as it is possible for mechanisms denoting a direct action of GF on the clock to occur simultaneously with those of a cell cycle influence on the clock.

Moreover, we have explored cell cycle period control, with relevance for cancer therapies, by means of single-parameter changes in the clock system. We have found that both slowing down and speeding up the clock allow to slow down the cell cycle: the first method by promoting a state of 1:1 synchronization and the second by accelerating the clock enough to drive the system away from the 1:1 region, which causes the cell cycle to adapt by slowing down and maintain a rational ratio of synchronization.

These methods may be relevant in the context of cancer therapies. However, as 
with the case of regular chemotherapies, the issue of cell heterogeneities may cause a part of the cell population to always remain resistant to the drug application. In this regard, Almeida et al. ${ }^{10}$ have shown that the phase of the clock/cell cycle is a control parameter for the system's synchronization response, i.e. the time of an input application determines whether a cell will be responsive or resistant to the input. Thus, further studies concerning the phase of these oscillators at the time of drug application may be a path to devise methods of increasing the number of responding cells, as well as of further developing chronotherapies. On this matter, while the phase of the clock would be a more important parameter to adjust in our studies of period control, the phase of the cell cycle is likely to be more relevant for apoptosis-inducing chemotherapies. Broadly, the methods developed in the present work may inform the creation of in vitro protocols for the control of the mammalian clock and cell cycle synchronization dynamics.

\section{Acknowledgements}

The authors are part of the Labex SIGNALIFE Network for Innovation on signal Transduction Pathways in Life Sciences (ANR-11-LABX-0028-01) and of the ICycle Project (ANR-16-CE33-0016-01).

\section{References}

1. Ozturk N, Ozturk D, Kavakli IH, Okyar A. Molecular Aspects of Circadian Pharmacology and Relevance for Cancer Chronotherapy. Int J Mol Sci. 2017 Oct 17;18(10).

2. Leproult R, Holmbck U, Van Cauter E. Circadian misalignment augments markers of insulin resistance and inflammation, independently of sleep loss. Diabetes. 2014 Jun;63(6):1860-9.

3. Fu L, Pelicano H, Liu J, Huang P, Lee C. The circadian gene Period2 plays an important role in tumor suppression and DNA damage response in vivo. Cell. 2002 Oct 4;111(1):41-50.

4. Prez-Roger I, Solomon DL, Sewing A, Land H. Myc activation of cyclin E/Cdk2 kinase involves induction of cyclin E gene transcription and inhibition of p27(Kip1) binding to newly formed complexes. Oncogene. 1997 May 22;14(20):2373-81.

5. Grchez-Cassiau A, Rayet B, Guillaumond F, Teboul M, Delaunay F. The circadian clock component BMAL1 is a critical regulator of p21WAF1/CIP1 expression and hepatocyte proliferation. J Biol Chem. 2008 Feb 22;283(8):4535-42.

6. Matsuo T, Yamaguchi S, Mitsui S, Emi A, Shimoda F, Okamura H. Control mechanism of the circadian clock for timing of cell division in vivo. Science. 2003 Oct $10 ; 302(5643): 255-9$.

7. Shostak A. Circadian Clock, Cell Division, and Cancer: From Molecules to Organism. Int J Mol Sci. 2017 Apr 20;18(4).

8. Feillet C, Krusche P, Tamanini F, Janssens RC, Downey MJ, Martin P, Teboul M, Saito S, Lvi FA, Bretschneider T, van der Horst GT, Delaunay F, Rand DA. Phase locking and multiple oscillating attractors for the coupled mammalian clock and cell cycle. Proc Natl Acad Sci U S A. 2014 Jul 8;111(27):9828-33.

9. Bieler J, Cannavo R, Gustafson K, Gobet C, Gatfield D, Naef F. Robust synchronization of coupled circadian and cell cycle oscillators in single mammalian cells. Mol Syst Biol. 2014 Jul 15;10:739. 
10. Almeida S, Chaves M, Franck D. Control of synchronization ratios in clock/cell cycle coupling by growth factors and glucocorticoids. R. Soc. open sci. 2020; 7:192054.

11. Nagoshi E, Saini C, Bauer C, Laroche T, Naef F, Schibler U. Circadian gene expression in individual fibroblasts: cell-autonomous and self-sustained oscillators pass time to daughter cells. Cell. 2004 Nov 24;119(5):693-705.

12. Almeida S, Chaves M, Franck D. Period Control of Coupled Clock and Cell Cycle Systems. Procceedings of CSBio 2019.

13. Zmborszky J, Hong CI, Csiksz Nagy A. Computational analysis of mammalian cell division gated by a circadian clock: quantized cell cycles and cell size control. J Biol Rhythms. 2007 Dec;22(6):542-53.

14. Grard C, Goldbeter A. Entrainment of the Mammalian Cell Cycle by the Circadian Clock: Modeling Two Coupled Cellular Rhythms. PLoS Comput Biol. 2012 May;8(5):e1002516.

15. Traynard P, Feillet C, Soliman S, Delaunay F, Fages F. Model-based investigation of the circadian clock and cell cycle coupling in mouse embryonic fibroblasts: Prediction of RevErb- $\alpha$ up-regulation during mitosis. Biosystems. 2016 Nov;149:59-69.

16. Yan J, Shi G, Zhang Z, Wu X, Liu Z, Xing L, Qu Z, Dong Z, Yang L, Xu Y. An intensity ratio of interlocking loops determines circadian period length. Nucleic Acids Res. 2014;42(16):10278-87.

17. Lee Y, Dominy JE, Choi YJ, Jurczak M, Tolliday N, Camporez JP, Chim H, Lim JH, Ruan HB, Yang X, Vazquez F, Sicinski P, Shulman GI, Puigserver P. .Cyclin D1-Cdk4 controls glucose metabolism independently of cell cycle progression. Nature. 2014 Jun $26 ; 510(7506): 547-51$

18. Almeida S, Chaves M, Delaunay F. Transcription-based circadian mechanism controls the duration of molecular clock states in response to signaling inputs. J Theor Biol. 2020; 484:110015.

19. Almeida S, Chaves M, Delaunay F, Feillet C. A comprehensive reduced model of the mammalian cell cycle. IFAC-PapersOnLine 2017; 50(1):12617-22.

20. Ye $\mathrm{P}, \mathrm{Hu} \mathrm{Q}$, Liu H, Yan Y, D'ercole AJ. beta-catenin mediates insulin-like growth factor-I actions to promote cyclin D1 mRNA expression, cell proliferation and survival in oligodendroglial cultures. Glia. 2010 Jul;58(9):1031-41

21. Bak P. The Devil's Staircase Physics Today. 1986 Dec;39(12):38.

22. Wallach T, Kramer A. Chemical chronobiology: Toward drugs manipulating time. FEBS Lett. 2015 Jun 22;589(14):1530-8.

23. Hirota T, Lewis WG, Liu AC, Lee JW, Schultz PG, Kay SA. A chemical biology approach reveals period shortening of the mammalian circadian clock by specific inhibition of GSK-3 $\beta$. Proc Natl Acad Sci U S A. 2008 Dec 30;105(52):20746-51.

24. Yin L, Wang J, Klein PS, Lazar MA. Nuclear receptor Rev-erb $\alpha$ is a critical lithiumsensitive component of the circadian clock. Science. 2006 Feb 17;311(5763):1002-5.

25. Fu L, Lee CC. The circadian clock: pacemaker and tumour suppressor. Nat Rev Cancer. 2003 May;3(5):350-61. 